\title{
Effectiveness and safety of hypotension fluid resuscitation in traumatic hemorrhagic shock: A systematic review and meta-analysis of randomized controlled trials
}

\author{
Kamil Safiejko ${ }^{1}$, Jacek Smereka ${ }^{2,3}$, Krzysztof J. Filipiak ${ }^{4}$, Agnieszka Szarpak ${ }^{5}$, \\ Marek Dabrowski ${ }^{6}$, Jerzy R. Ladny ${ }^{3,7}$, Milosz J. Jaguszewski ${ }^{8}$, Lukasz Szarpak ${ }^{3,9}$ \\ ${ }^{1}$ Comprehensive Cancer Center in Bialystok, Poland \\ ${ }^{2}$ Department of Emergency Medical Service, Wroclaw Medical University, Wroclaw, Poland \\ ${ }^{3}$ Polish Society of Disaster Medicine, Warsaw, Poland \\ ${ }^{4}$ First Chair and Department of Cardiology, Medical University of Warsaw, Poland \\ ${ }^{5}$ Maria Skłodowska-Curie Medical Academy in Warsaw, Poland \\ ${ }^{6}$ Chair and Department of Medical Education, Poznan University of Medical Sciences, Poznan, Poland \\ ${ }^{7}$ Clinic of Emergency Medicine, Medical University of Bialystok, Poland \\ ${ }^{8}$ First Department of Cardiology, Medical University of Gdansk, Poland \\ ${ }^{9}$ Bialystok Oncology Center, Bialystok, Poland
}

\section{This paper was guest edited by Prof. Togay Evrin}

\begin{abstract}
Background: Although the resuscitation of an adult trauma patient has been researched and written about for the past century, the ideal fluid strategy to infuse during the initial resuscitation period remains unresolved. This work was aimed at assessing the effect of hypotensive versus conventional resuscitation strategies in traumatic hemorrhagic shock patients on mortality, and the need for blood transfusions including adverse events.

Methods: This systematic review and meta-analysis were performed following the PRISMA guidelines. Electronic databases were searched for randomized controlled trials (RCT) comparing the effect of hypotension versus conventional fluid resuscitation for traumatic hemorrhagic shock patients. Two reviewers independently performed the screening, data extraction, and bias assessment. The data analysis was completed using the Cochrane Collaboration's software RevMan 5.4.

Results: Data from 28 RCTs on 4503 patients were included in the final meta-analysis. Patients receiving hypotension fluid resuscitation compared with conventional fluid resuscitation experienced less mortality (12.5\% vs. $21.4 \% ; R R=0.58 ; 95 \%$ CI: 0.51-0.66; $p<0.001)$, fewer adverse events $(10.8 \%$ vs. $13.4 \% ; R R=0.70 ; 95 \%$ CI: 0.59-0.83; $p<0.001$ ), including fever acute respiratory distress syndrome (7.8\% vs. $16.8 \%)$ or multiple organ dysfunction syndrome (8.6\% vs. $21.6 \%)$.

Conclusions: This meta-analysis showed that hypotensive fluid resuscitation significantly reduced the mortality of hypovolemic shock patients. Findings are low in certainty and should be interpreted with caution. Therefore, there is an urgent need for larger, multicenter, randomized trials to confirm these findings. (Cardiol J 2022; 29, 3: 463-471)
\end{abstract}

Key words: fluid resuscitation, restricted fluid resuscitation, hemorrhagic shock, hemorrhage, meta-analysis, systematic review

Address for correspondence: Lukasz Szarpak, Assoc. Prof. PhD, Bialystok Oncology Center, ul. Ogrodowa 12, 15-027 Białystok, Poland, tel: +48 500186225, e-mail: lukasz.szarpak@gmail.com

Received: 25.06.2020 Accepted: 6.07.2020 Early publication date: 10.07.2020

This article is available in open access under Creative Common Attribution-Non-Commercial-No Derivatives 4.0 International (CC BY-NC-ND 4.0) license, allowing to download articles and share them with others as long as they credit the authors and the publisher, but without permission to change them in any way or use them commercially. 


\section{Introduction}

Trauma injury remains the leading cause of death among people aged less than 35 years, with $40 \%$ of trauma deaths imputable to uncontrolled hemorrhagic shock or its consequences [1,2].

Currently, fluid resuscitation is the first step in the hemodynamic management of traumatic hemorrhagic shock [3]. The rapid vascular access and stabilization of the cardiovascular system can protect the patient from the severe consequences of hypovolemic shock. The origins of fluid resuscitation can be traced back to the thirties of the nineteenth century, when Thomas Latta performed an attempt of intravenous fluid resuscitation for the first time [4]. In the period 1879-1881 Kroneecker and Landerer stated that in cases of blood loss the most valuable thing is to rapidly restore the vascular bed volume. For this purpose, they proposed using a normal saline solution with the addition of sugar $[5,6]$. The development of fluid therapy was in the 1920s, when Alfred Blalock experimented with incremental hemorrhage to induce shock in dogs $[7,8]$. In his research, Blalock used blood pressure (BP), cardiac output as well as blood oxygen content from left and right ventricles to evaluate the effect of three types of treatment: saline, transfusion and pharmacological treatment.

Applying an appropriate fluid therapy strategy may restore tissue perfusion and consequently oxygenation of the body. Fluid resuscitation can be carried out based on changes in hemodynamics, diuresis, serum lactate levels or alkaline deficit. However, excessive fluid resuscitation could contribute to the development of coagulopathy of trauma [3, 9] as well as tissue edema [10], which can lead to alterations of tissue perfusion and complications such as abdominal compartment syndrome or adult respiratory distress syndrome $[11,12]$. The optimal level of BP during resuscitation of hemorrhagic shock patients is still debated.

The present work aimed to assess the effect of hypotensive versus conventional resuscitation strategies in traumatic hemorrhagic shock patients on mortality, need for blood transfusion and adverse events (specifically: acute myocardial or renal failure or acute respiratory distress syndrome).

\section{Methods}

This systematic review and meta-analysis adhere to the reporting guidelines of the Preferred Reporting Items for Systematic reviews and Metaanalyses (PRISMA) statement (Suppl. Table 1).

\section{Search strategy}

Available literature databases including EMBASE, MEDLINE, Google Scholar, Cochrane Central Register of Controlled Trials (CENTRAL) were searched from the inception of the databases until 18 June 2020. Searches were conducted independently by two persons (K.S. and L.S.). The papers were restricted to those with the English language. Reference lists of eligible articles were reviewed and content experts were consulted with (K.J.F. and M.J.J.) to identify additional published reports. Incomplete data were dealt with by contacting the principal authors, when possible, to ask for missing or unclear information.

The search strategy was comprised of MESH terms and keywords such as: "shock"OR, "hemorrhagic" OR, "trauma” OR, "injury” OR, "hypotensive resuscitation" OR, "limited resuscitation" OR, "fluid resuscitation" OR, "limited fluid". To identify in-progress or terminated studies, clinicaltrials.gov registry was also searched.

\section{Study selection}

This study included randomized controlled trials (RCTs) and quasi-randomized trials. Observational studies, case reports, studies not based on original research and studies not involving patients, conference papers as well as letters to the editor were excluded from the present study.

\section{Data extraction}

Using a standardized data extraction sheet, two authors (K.S. and L.S.) independently extracted data from each report included. Any discrepancies were resolved by consensus with the third author (J.S.). When necessary for data or article clarification, personal communication was made with select study authors. Baseline patient characteristics were extracted as well as data about each trial's intervention, inclusion and exclusion criteria, mortality and adverse events. For all clinical outcomes, the number of events that occurred in each arm of each trial were tabulated.

\section{Quality assessment}

Two reviewers (J.S. and A.S.) independently assessed the methodological quality of each eligible article using the "risk of bias" assessment tool of the Cochrane Handbook [13]. The following domains were evaluated for RCTs: random sequence generation (selection bias), allocation concealment (selection bias), blinding of participants and personnel (performance bias), blinding of outcome assessment (detection bias), incomplete 
outcome data (attrition bias), selective reporting (reporting bias) and other bias [12]. Each was graded "yes", "no", or "unclear", which reflected a high risk of bias, low risk of bias, and uncertain bias, respectively (Suppl. Fig. S1). The review authors' judgments about each risk of bias item are provided in Supplementary Figure S2. The overall risk of bias for the study was rated 'low' if 7 or more domains were rated low, 'moderate' if 4 to 6 domains were rated low, and 'high' if 1 to 3 domains were rated low.

\section{Statistical analysis}

Statistical analysis was done by two authors (A.S. and L.S.) independently and was crossvalidated. For continuous outcomes, mean difference (MD), and for dichotomous outcomes were used, and risk ratios (RR), were calculated. All continuous data with either means with standard deviations (SD) or medians with interquartile ranges (IQR) as reported in the primary study are presented. When the continuous outcome was reported in a study as median, range, and IQR, means and SD using the formula described by Hozo et al. [14] were estimated. For descriptive purposes, absolute and relative frequencies are reported for categoric variates.

Statistical heterogeneity and inconsistency were measured by using the Cochran $\mathrm{Q}$ test and $\mathrm{I}^{2}$, respectively [14]. Odds ratios (OR) with $95 \%$ confidence intervals (CI) were calculated as summary statistics. The pooled OR was calculated with the Mantel-Haenszel method. Weighted mean differences and $95 \%$ CIs were computed for continuous variables, again using a fixed-effect method in cases of low statistical inconsistency $\left(\mathrm{I}^{2} \leq 50 \%\right)$ and using a random-effect method in cases of moderate or high statistical inconsistency $\left(\mathrm{I}^{2}>50 \%\right)[15]$. Results were considered statistically significant at $\mathrm{p}<0.05$. Statistical analyses were performed with the Review Manager (version 5.4; The Nordic Cochrane Centre, The Cochrane Collaboration, Copenhagen, Denmark).

\section{Results}

\section{Eligible studies and their characteristics}

The flowchart of the literature search is presented in Figure 1. The initial search returned 432 records from all the databases. Two more studies were identified from the references of the relevant articles. Two hundred and ninety-six records were further screened by titles and abstracts and $138 \mathrm{du}-$ plicate articles were removed. Unrelated articles, comments, reviews, letters, and duplicate articles were excluded. Then 47 articles were assessed by accessing the full-text. Nineteen studies were excluded because of unavailable data, duplicates, and unrelated topics. Finally, 28 studies were included in the analysis [16-43].

\section{Assessment of quality}

The quality assessment is represented in Supplementary Figures S1 and S2. The method of random sequence generation was perfect in all the studies. There were high risks of attrition bias, lack of intention-to-treat analysis, and selective reporting. The detailed information about blinding and allocation concealment was insufficient in most studies. None of the trials included was at low risk of bias across all domains.

\section{Mortality}

Twenty-eight studies reported overall mortality [16-23]. Mortality with hypotension fluid resuscitation was $12.5 \%$ and was statistically significant, being smaller than with the conventional fluid resuscitation group $-21.4 \%(\mathrm{RR}=0.58 ; 95 \% \mathrm{CI}$ : 0.51-0.66; $\mathrm{I}^{2}=37 \% ; \mathrm{p}<0.001$; Fig. 2). In contrast, only one study by Morrison et al. [28] indicated mortality rates during the first 24 hours. According to this study, mortality for hypotension versus conventional fluid resuscitation varied and amounted to $13.6 \%$ vs. $21.7 \%$ respectively $(\mathrm{RR}=0.63$; 95\% CI: 0.25-1.58; $\mathrm{p}=0.32$ ).

\section{Adverse events}

The polled analysis showed that hypotension fluid resuscitation compared to conventional fluid resuscitation was associated with a lower risk of adverse events (10.8\% vs. $13.4 \%$, respectively; $\mathrm{RR}=0.70 ; 95 \%$ CI: 0.59-0.83; $\left.\mathrm{I}^{2}=52 \% ; \mathrm{p}<0.001\right)$.

The use of hypotension versus conventional fluid resuscitation showed a higher incidence of anemia ( $74.3 \%$ vs. $68.6 \%)$, thrombocytopenia $(33.6 \%$ vs. $29.4 \%)$ and acute renal failure $(8.8 \%$ vs. $8.1 \%)$. In other types of adverse events the relationship was reversed, and the use of hypotension fluid resuscitation was associated with a lower risk of complications (Table 1).

Fluid balance and transfusion requirements

Additional analysis showed that patients who were treated with hypotension fluid resuscitation required smaller volumes of fluids than the conventional fluid resuscitation group ( $\mathrm{MD}=-1.02 ; 95 \%$ CI: -1.33 to $-0.71 ; \mathrm{I}^{2}=99 \%$; $<0.001$; Fig. 3 ). 


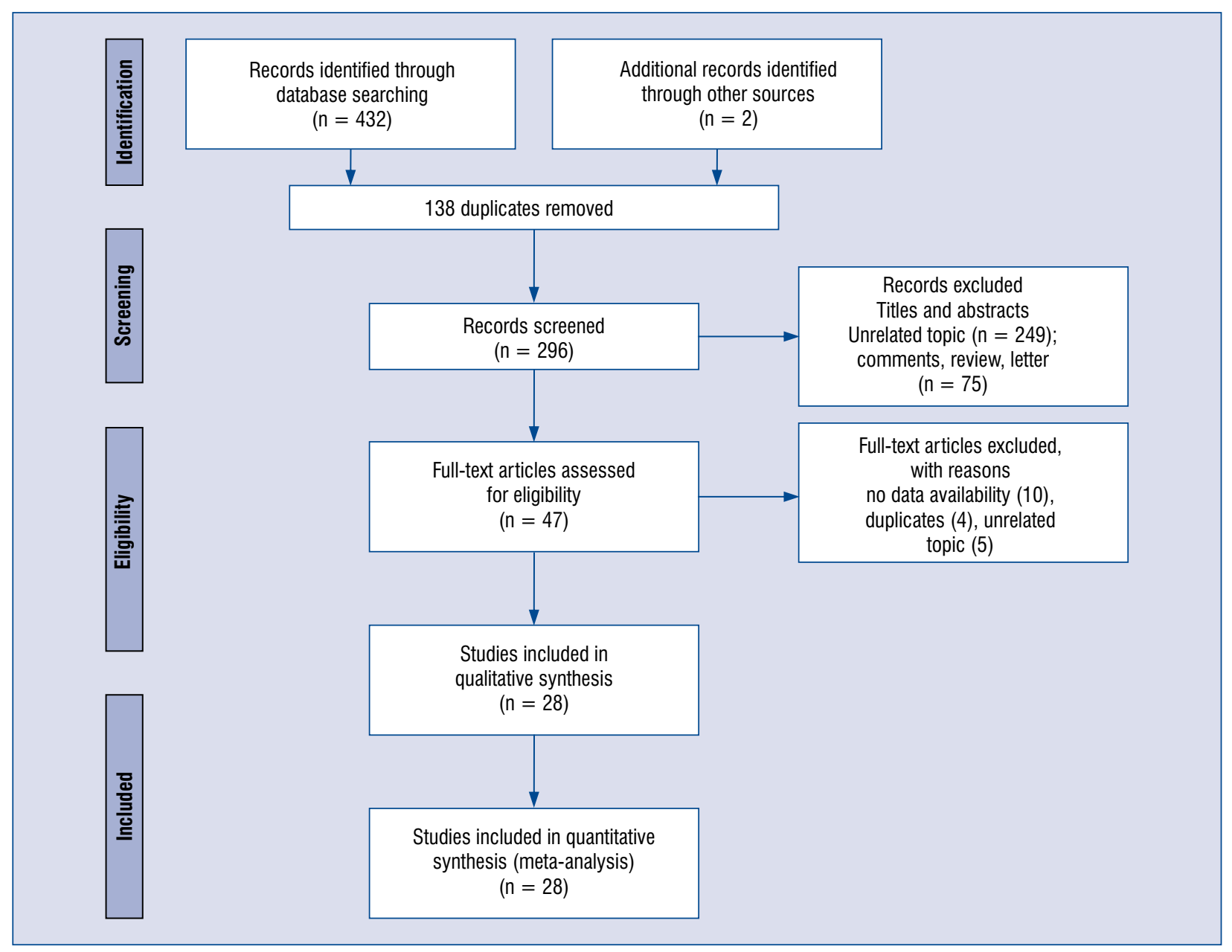

Figure 1. Flow diagram showing stages of database searching and study selection as per PRISMA guidelines.

\section{Length of stay ICU/hospital}

The length of stay in the intensive care unit (ICU) was reported by two studies [16, 32]. The polled analysis did not show significant differences in the length of stay in ICU between the groups $\left(\mathrm{MD}=0.38 ; 95 \% \mathrm{CI}:-1.83-2.59 ; \mathrm{I}^{2}=73 \% ; \mathrm{p}=\right.$ $=0.74$; Suppl. Fig. S3). Three studies indicated length of stay in hospital [16, 29, 32]. The difference between therapeutic groups was not statistically significant $(\mathrm{MD}=-0.82 ; 95 \% \mathrm{CI}:-2.43-0.78$; $\mathrm{I}^{2}=0 \% ; \mathrm{p}=0.32$; Suppl. Fig. S4).

\section{Discussion}

The purpose of this research was to compare the effects of hypovolemic and conventional fluid resuscitation on the mortality rate among patients with traumatic hemorrhagic shock. Meta-analysis for overall mortality showed that hypovolemic fluid resuscitation offered benefit in comparison with conventional fluid resuscitation for patients with traumatic hemorrhagic shock at the final follow-up $(\mathrm{p}<0.001)$.

Obtaining intravascular access in hypovolemic patients (especially trauma patients) should be done as soon as possible. In patients with hemorrhage, the most important part of the procedure is to stop the hemorrhage. In such a patient's hospital setting, transfusion of blood substitutes should be limited in favor of the transfusion of blood components. It is recommended to transfuse the red blood cell concentrate in a volume that maintains the hemoglobin concentration at 7-9 g/dL. Fresh frozen plasma should be transfused immediately at a dose of 10 $-15 \mathrm{~mL} / \mathrm{kg}$ b.w. and further replenishment of fresh frozen plasma should depend on the volume of red blood cells transfused and the coagulogram. In the case of platelets, they should be transfused in sufficient quantities to maintain a concentration of 50,000 / $/ \mathrm{mL}$. To mitigate the effects of hypovolemic shock caused by the injury, transfusion of cryoprecipitate or fibrinogen concentrate may also be considered. 


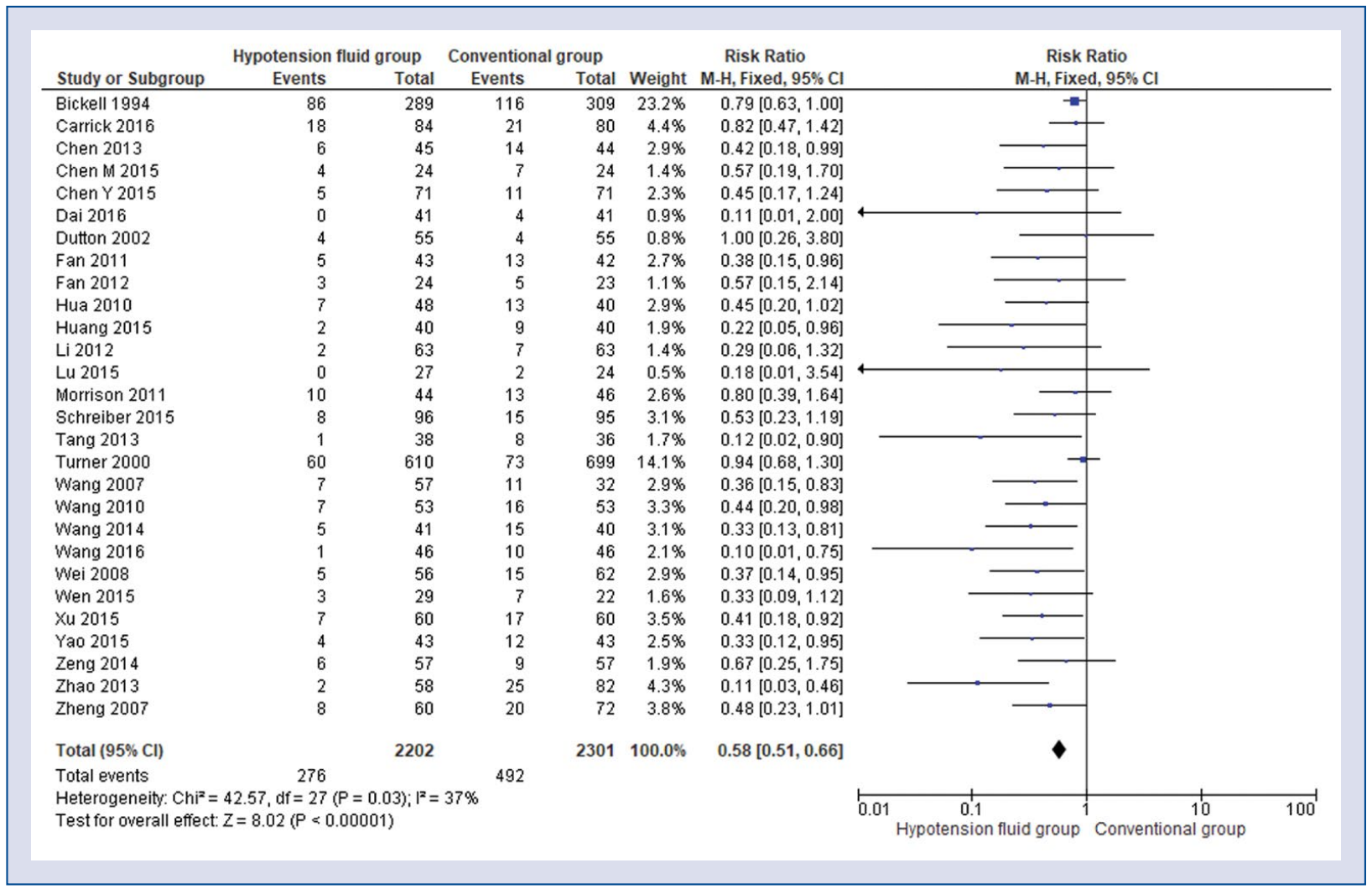

Figure 2. Forest plot of hypotension versus. conventional fluid resuscitation, relative to mortality. The center of each square represents the relative risk for individual trials, and the corresponding horizontal line stands for a $95 \%$ confidence interval (CI). The diamonds represent pooled results.

Table 1. Comparison of hypotension and conventional fluid resuscitation relative to adverse events.

\begin{tabular}{lcccccc}
\hline & $\begin{array}{c}\text { Number } \\
\text { of trials }\end{array}$ & $\begin{array}{c}\text { Hypotension } \\
\text { fluid } \\
\text { resuscitation }\end{array}$ & $\begin{array}{c}\text { Conventional } \\
\text { fluid } \\
\text { resuscitation }\end{array}$ & $\begin{array}{c}\text { RR or MD } \\
\text { (95\% CI) }\end{array}$ & $\begin{array}{c}\text { P } \\
\text { value }\end{array}$ & $\begin{array}{c}\mathbf{I}^{2} \\
\text { statistic, } \\
\%\end{array}$ \\
\hline ARDS & 13 & $7.8 \%$ & $16.8 \%$ & $0.44[0.34-0.58]$ & $<0.001$ & $0 \%$ \\
Acute myocardial infarction & 1 & $1.3 \%$ & $1.5 \%$ & $0.88[0.06-13.79]$ & 0.93 & - \\
Stroke & 1 & $0 \%$ & $3.0 \%$ & $0.18[0.01-3.61]$ & 0.26 & - \\
Sepsis syndrome & 2 & $3.5 \%$ & $3.9 \%$ & $0.91[0.42-1.98]$ & 0.82 & $0 \%$ \\
MODS & 10 & $8.6 \%$ & $21.6 \%$ & $0.42[0.30-0.60]$ & $<0.001$ & $0 \%$ \\
Any renal failure & 1 & $14.7 \%$ & $12.1 \%$ & $1.21[0.52-2.83]$ & 0.66 & - \\
Acute renal failure & 8 & $8.8 \%$ & $8.1 \%$ & $0.99[0.53-1.86]$ & 0.98 & $61 \%$ \\
Anemia & 2 & $74.3 \%$ & $68.6 \%$ & $1.11[0.96-1.28]$ & 0.16 & $2 \%$ \\
Hypotension & 1 & $13.3 \%$ & $16.7 \%$ & $0.80[0.36-1.76]$ & 0.58 & - \\
Coagulopathy & 3 & $15.7 \%$ & $15.8 \%$ & $0.95[0.73-1.24]$ & 0.73 & $0 \%$ \\
Thrombocytopenia & 2 & $33.6 \%$ & $29.4 \%$ & $1.21[0.64-2.28]$ & 0.56 & $54 \%$ \\
Pneumonia & 1 & $7.6 \%$ & $9.1 \%$ & $0.84[0.49-1.43]$ & 0.52 & - \\
Deterioration in T-RTS & 1 & $7.4 \%$ & $7.9 \%$ & $0.93[0.50-1.71]$ & 0.81 & - \\
Complications not specified & 1 & $7.5 \%$ & $8.6 \%$ & $0.88[0.61-1.27]$ & 0.49 & - \\
\hline
\end{tabular}

ARDS - acute respiratory distress syndrome; MORS — multiple organ dysfunction syndrome; MD — mean difference; RR — risk ratio 


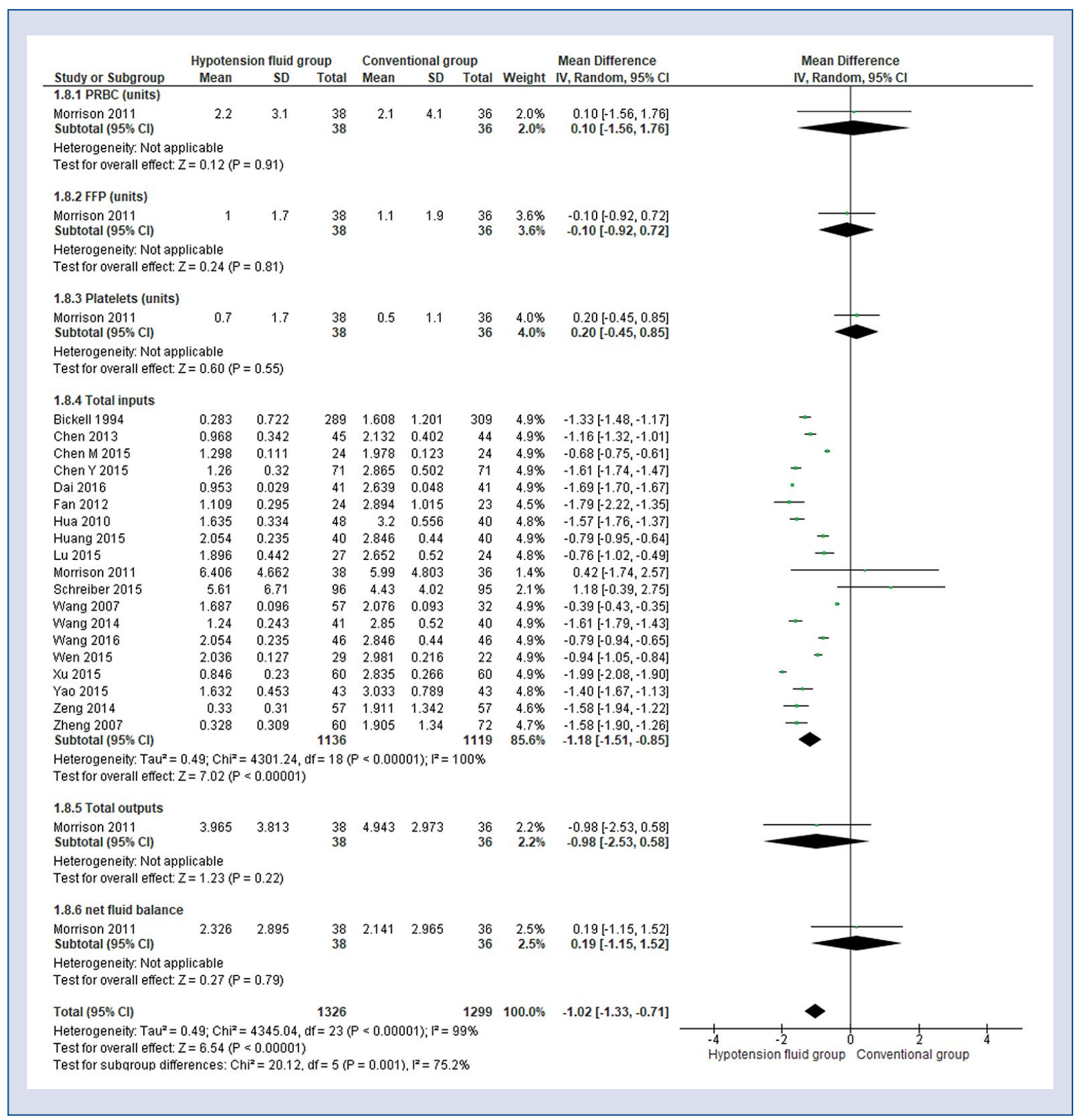

Figure 3. Forest plot of hypotension versus conventional fluid resuscitation, relative to fluid balance and transfusion requirements. The center of each square represents the mean difference for individual trials, and the corresponding horizontal line stands for $95 \%$ confidence interval $(\mathrm{Cl})$. The diamonds represent pooled results.

Fluid therapy aims not only to maintain and restore the intravascular volume but also, by optimizing the preload, to increase cardiac output and improve tissue perfusion. Discussions are still ongoing as to whether crystalloids (i.e. $0.9 \%$ saline or Ringer's Lactate) or colloidal solutions (i.e. dextran's, gelatins, HAES) should be used in the initial phase of fluid resuscitation. De Crescenzo et al. [44] indicates no beneficial effect of hyper- tonic saline with or without dextran in general trauma patients. In turn, Martin et al. [45] in his meta-analysis indicated that crystalloids were less efficient than colloids at stabilizing resuscitation endpoints. The application of an appropriate fluid resuscitation strategy is, therefore, more important than the type of fluid administered. Malbrain et al. [46] showed that a positive cumulative fluid balance is associated with intra-abdominal 
hypertension and worse outcomes. Hypotensive fluid resuscitation as shown by numerous studies may offer a survival benefit over conventional fluid resuscitation for trauma patients. It can also further reduce blood loss and thus blood product utilization. It can, therefore, be concluded that fluid resuscitation in trauma patients should be based on a specific compromise between too small a volume leading to hypoperfusion and too much hydration in patients, which may result in increased bleeding due to increased BP. The use of too large a volume of fluid can lead to "dilutive" hemorrhagic bleeding.

As indicated by numerous randomized studies — as also confirmed by this meta-analysis - hypovolemic fluid resuscitation can bring benefits in the management of the trauma patient [28, 29, 47]. However, it should be remembered that the hypotension should not last longer than 1 hour $[22,28]$. It should be kept in mind that the rules of fluid resuscitation in trauma patients with concomitant craniocerebral trauma are different. Thereafter, as recommended by the National Institute for Health and Clinical Excellence (NICE), it is important to increase BP (systolic BP $110-120 \mathrm{mmHg}$ ) as quickly as possible to secure proper brain perfusion and prevent secondary brain changes.

In this meta-analysis, hypovolemic fluid resuscitation was associated with a higher incidence of thrombocytopenia, renal failure or anemia in comparison with conventional management. The above symptoms are closely related. Thrombocytopenia is the most frequently diagnosed hemorrhagic flaw and may lead to anemia. As many authors indicate, acute kidney injury is a common feature in patients with thrombocytopeniaassociated multiple organ failure with incidences as high as $42 \%$ in disseminated intravascular coagulation, $58 \%$ in thrombocytopenic purpura and $100 \%$ in hemolytic uremic syndromes [48-50]. On the other hand, in the case of conventional fluid resuscitation, a statistically significantly higher incidence of acute respiratory distress syndrome (ARDS) or multiple organ dysfunction syndrome (MODS) was observed. Jiang et al. [51] indicate that hypovolemic/restricted fluid resuscitation can effectively eliminate inflammatory factors, improve immune function, maintain the stability of blood components, and reduce the incidences of ARDS and MODS. In the case of hypotension fluid resuscitation, there was also a higher incidence of acute myocardial infarction, which may be caused by lower myocardial overload.

\section{Limitations of the study}

The present study has some limitations. First, only in several articles study groups are appropriate, in other articles the sample size is relatively small, which led to a wide $95 \%$ CI. Second, two studies referred to mean arterial pressure, the others to systolic blood pressure.

\section{Conclusions}

The present study findings show significant associations between hypotensive fluid resuscitation and a decreased risk of adverse events and cardiovascular mortality in hypovolemic shock trauma patients. There is an urgent need for a large multicenter randomized trial to confirm these findings.

\section{Acknowledgments}

The study was supported by the ERC Research Net and by the Polish Society of Disaster Medicine.

Conflict of interest: None declared

\section{References}

1. Alberdi F, García I, Atutxa L, et al. Trauma and Neurointensive Care Work Group of the SEMICYUC. Epidemiology of severe trauma. Med Intensiva. 2014; 38(9): 580-588, doi: 10.1016/j. medin.2014.06.012, indexed in Pubmed: 25241267.

2. Alexandrescu R, O'Brien SJ, Lecky FE. A review of injury epidemiology in the UK and Europe: some methodological considerations in constructing rates. BMC Public Health. 2009; 9: 226-231, doi: 10.1186/1471-2458-9-226, indexed in Pubmed: 19591670.

3. Krzych ŁJ, Czempik PF. Effect of fluid resuscitation with balanced solutions on platelets: In vitro simulation of $20 \%$ volume substitution. Cardiol J. 2018; 25(2): 254-259, doi: 10.5603/ CJ.a2017.0054, indexed in Pubmed: 28497841.

4. Baskett T. William O'Shaughnessy, Thomas Latta and the origins of intravenous saline. Resuscitation. 2002; 55(3): 231-234, doi: 10.1016/s0300-9572(02)00294-0, indexed in Pubmed: 12458058.

5. Milano R. Fluid resuscitation of the adult trauma patient: where have we been and where are we going? Nurs Clin North Am. 2017; 52(2): 237-247, doi: 10.1016/j.cnur.2017.01.001, indexed in Pubmed: 28478872.

6. Kalkwarf KJ, Cotton BA. Resuscitation for hypovolemic shock. Surg Clin North Am. 2017; 97(6): 1307-1321, doi: 10.1016/j. suc.2017.07.011, indexed in Pubmed: 29132511.

7. Beaty CA, George TJ, Conte JV. Historical perspectives of The American Association for Thoracic Surgery: Alfred Blalock (1899-1964). J Thorac Cardiovasc Surg. 2012; 143(2): 260-263, doi: 10.1016/j.jtcvs.2011.10.071, indexed in Pubmed: 22248679.

8. Ravitch MM. The contributions of Alfred Blalock to the science and practice of surgery. Johns Hopkins Med J. 1977; 140(2): 57-67, indexed in Pubmed: 319290. 
9. Srivastava A. Fluid resuscitation: principles of therapy and. Adv Chronic Kidney Dis. 2017; 24(4): 205-212, doi: 10.1053/j. ackd.2017.05.002, indexed in Pubmed: 28778359.

10. Solomonov E, Hirsh M, Yahiya A, et al. The effect of vigorous fluid resuscitation in uncontrolled hemorrhagic shock after massive splenic injury. Crit Care Med. 2000; 28(3): 749-754, doi: 10.1097/00003246-200003000-00024, indexed in Pubmed: 10752825.

11. Varela JE, Cohn SM, Diaz I, et al. Splanchnic perfusion during delayed, hypotensive, or aggressive fluid resuscitation from uncontrolled hemorrhage. Shock. 2003; 20(5): 476-480, doi: 10.1097/01.SHK.0000094036.09886.9b, indexed in Pubmed: 14560114.

12. Muttath A, Annayappa Venkatesh L, Jose J, et al. Adverse outcomes due to aggressive fluid resuscitation in children: a prospective observational study. J Pediatr Intensive Care. 2019; 8(2): 64-70, doi: 10.1055/s-0038-1667009, indexed in Pubmed: 31093457.

13. Higgins JPT, Green S, editors. Cochrane handbook for systematic reviews of interventions. Version 5.1 [updated March 2011]. The Cochrane Collaboration. http://handbook-5-1.cochrane.org/ (Accessed 10 June 2020).

14. Hozo SP, Djulbegovic B, Hozo I. Estimating the mean and variance from the median, range, and the size of a sample. BMC Med Res Methodol. 2005; 5: 13, doi: 10.1186/1471-2288-5-13, indexed in Pubmed: 15840177.

15. Higgins JPT, Thompson SG, Deeks JJ, et al. Measuring inconsistency in meta-analyses. BMJ. 2003; 327(7414): 557-560, doi: 10.1136/bmj.327.7414.557, indexed in Pubmed: 12958120.

16. Bickell WH, Wall MJ, Pepe PE, et al. Immediate versus delayed fluid resuscitation for hypotensive patients with penetrating torso injuries. N Engl J Med. 1994; 331(17): 1105-1109, doi: 10.1056/NEJM199410273311701, indexed in Pubmed: 7935634.

17. Carrick MM, Morrison CA, Tapia NM, et al. Intraoperative hypotensive resuscitation for patients undergoing laparotomy or thoracotomy for trauma: Early termination of a randomized prospective clinical trial. J Trauma Acute Care Surg. 2016; 80(6): 886-896, doi: 10.1097/TA.0000000000001044, indexed in Pubmed: 27015578.

18. Chen M, Zhong W, Hu Y. The study on limited fluid resuscitation patients with traumatic shock. Sichuan Med J. 2013; 34: 1-10.

19. Chen M. Analysis on the efficacy of limited fluid resuscitation of traumatic hemorrhagic shock. China Academic J. 2015; 22: 3-6.

20. Chen I, Bin L, Ai-guo Z, et al. The application of limited fluid resuscitation in first aid of hemorrhagic traumatic shock. J Hunan Normal Univ. 2015; 12: 1-5.

21. Dai Y. Effect of limited fluid resuscitation on prognosis of patients with severe closed traumatic hemorrhagic shock. J Liaoning Medical University. 2016; 37: 1-5.

22. Dutton RP, Mackenzie CF, Scalea TM. Hypotensive resuscitation during active hemorrhage: impact on in-hospital mortality. J Trauma. 2002; 52(6): 1141-1146, doi: 10.1097/00005373200206000-00020, indexed in Pubmed: 12045644.

23. Fun H, Yue M, Wu Y, et al. Early therapeutic effects of limited fluid resuscitation on severe pelvic fracture combined with hemorrhagic shock. J Traumatic Surg. 2011; 13(5): 1-4.

24. Fan H, Wu Y, Yue M. Early therapeutic effects of limited fluid resuscitation on severe hepatic and spleen injury combined with hemorrhagic shock. J Trauma Surg. 2012; 14: 10-16.

25. Hua LD, Tu YY, Fu JF, et al. Application of limited fluid resuscitation in patients with severe multiple trauma hemorrhagic shock. Progress in Modern Biomedicine. 2010; 10(13): 2462-2464.
26. Li W, Lin H, Deng X. Clinical discussion of limited fluid resuscitation on hemorrhagic traumatic shock without controlling bleeding. J Clini Med Engineering. 2012; 19: 1-9.

27. Lu Bo, Li MQ, Li JQ. The use of limited fluid resuscitation and blood pressure-controlling drugs in the treatment of acute upper gastrointestinal hemorrhage concomitant with hemorrhagic shock. Cell Biochem Biophys. 2015; 72(2): 461-463, doi: 10.1007/s12013-014-0487-2, indexed in Pubmed: 25567656.

28. Morrison CA, Carrick MM, Norman MA, et al. Hypotensive resuscitation strategy reduces transfusion requirements and severe postoperative coagulopathy in trauma patients with hemorrhagic shock: preliminary results of a randomized controlled trial. J Trauma. 2011; 70(3): 652-663, doi: 10.1097/ TA.0b013e31820e77ea, indexed in Pubmed: 21610356.

29. Schreiber MA, Meier EN, Tisherman SA, et al. A controlled resuscitation strategy is feasible and safe in hypotensive trauma patients: results of a prospective randomized pilot trial. J Trauma Acute Care Surg. 2015; 78(4): 687-95; discussion 695, doi: 10.1097/TA.0000000000000600, indexed in Pubmed: 25807399.

30. Huang T, Zhang Y. Analysis of the therapeutic effect of limited fluid resuscitation in hemorrhagic shock patients. China Modern Doctor. 2015; 53: 11-15.

31. Tang T, Li WP. The curative effect analysis of limited fluid resuscitation to esophageal variceal bleeding combined with hemorrhagic shock. Modern Practical Medicine. 2013; 25: 3-8.

32. Turner J, Nicholl J, Webber I, et al. A randomised controlled trial of prehospital intravenous fluid replacement therapy in serious trauma. Health Technology Assessment. 2000; 4(31), doi: 10.3310/hta4310.

33. Wang A, Gao J, Li X, et al. The significance of APACHE II and IL-6 in patients with traumatic hemorrhagic shock by limited fluids resuscitation. Tianjin Med J. 2010; 38(11): 1-6.

34. Wang Mt, Mei b, He J, et al. Effect of preoperative limited fluid resuscitation to the patients with traumatic shock. J Medi Coll PLA. 2007; 22(4): 226-229, doi: 10.1016/s1000-1948(07)60045-8.

35. Wang X, L Z, Wei L. Diagnosis and treatment of traumatic hemorrhagic shock caused by rupture of the liver and spleen on early fluid resuscitation. J Hepatobiliary Surg. 2014; 22: 4-7.

36. Wang F. Efficacy of limited fluid resuscitation in the early treatment of patients with active hemorrhagic shock. Henan Med Res. 2016; 25: 3-9.

37. Wei SK, He Y, Huang YQ, et al. Clinical analysis of 56 hemorrhagic patients with limited fluid resuscitation. J Youjiang National Med Coll. 2008; 1(2): 9.

38. Wen Z, Liu J, Chen J. Comparison of application effects by hypertonic saline fluid resuscitation, limited fluid resuscitation and conventional fluid resuscitation in traumatic hemorrhagic shock. China Prac Med. 2015; 10: 15-18.

39. Xu G. Clinical observation on limited fluid resuscitation in the treatment of uncontrolled hemorrhagic shock. Chinese J Disaster Med. 2015; 3: 5-9.

40. Yao J, Lu J. A comparative study on the clinical effect of limited fluid resuscitation and active fluid resuscitation in the treatment of patients with multiple trauma and hemorrhagic shock. Chinese J Frontier Med. 2015; 7: 6-10.

41. Zeng F, Deng Z, Hu M, et al. Clinical observation of limited fluid resuscitation on preoperative uncontrolled hemorrhagic shock. J Chin Pract Diagn Ther. 2014; 28: 1-5.

42. Zhao Y. The clinical effect analysis of limited fluids resuscitation in treatment of hemorrhagic shock. Medical Innovation of China. 2013; 10: 36-40. 
43. Zheng W, Wang X, Xu H. Effects of limited fluid resuscitation in treatment of hemorrhagic traumatic shock. China J Emergency Resuscitation Disaster Med. 2007; $2: 9$.

44. De Crescenzo C, Gorouhi F, Salcedo ES, et al. Prehospital hypertonic fluid resuscitation for trauma patients: A systematic review and meta-analysis. J Trauma Acute Care Surg. 2017; 82(5): 956-962, doi: 10.1097/TA.0000000000001409, indexed in Pubmed: 28257392.

45. Martin GS, Bassett P. Crystalloids vs. colloids for fluid resuscitation in the Intensive Care Unit: A systematic review and meta-analysis. J Crit Care. 2019; 50: 144-154, doi: 10.1016/j. jcrc.2018.11.031, indexed in Pubmed: 30540968.

46. Malbrain ML, Marik PE, Witters I, et al. Fluid overload, deresuscitation, and outcomes in critically ill or injured patients: a systematic review with suggestions for clinical practice. Anaesthesiol Intensive Ther. 2014; 46(5): 361-380, doi: 10.5603/ AIT.2014.0060, indexed in Pubmed: 25432556.

47. Hjortrup PB, Haase N, Bundgaard H, et al. Restricting volumes of resuscitation fluid in adults with septic shock after initial mana- gement: the CLASSIC randomised, parallel-group, multicentre feasibility trial. Intensive Care Med. 2016; 42(11): 1695-1705, doi: 10.1007/s00134-016-4500-7, indexed in Pubmed: 27686349.

48. Zafrani L, Mariotte E, Darmon M, et al. Acute renal failure is prevalent in patients with thrombotic thrombocytopenic purpura associated with low plasma ADAMTS13 activity. J Thrombosis Haemostasis. 2015; 13(3): 380-389, doi: 10.1111/jth.12826.

49. In JiW, Kim JE, Jeong JS, et al. Diagnostic and prognostic significance of neutrophil gelatinase-associated lipocalin in disseminated intravascular coagulation. Clin Chim Acta. 2014; 430: 145-149, doi: 10.1016/j.cca.2014.01.022, indexed in Pubmed: 24508993.

50. Levi M, van der Poll T. Disseminated intravascular coagulation: a review for the internist. Intern Emerg Med. 2013; 8(1): 23-32, doi: 10.1007/s11739-012-0859-9, indexed in Pubmed: 23015284.

51. Jiang LM, He J, Xi XY, et al. Effect of early restrictive fluid resuscitation on inflammatory and immune factors in patients with severe pelvic fracture. Chin J Traumatol. 2019; 22(6): 311-315, doi: 10.1016/j.cjtee.2019.07.008, indexed in Pubmed: 31685356 . 